\title{
The Influence of Boundary Condition on Numerical Simulation of Blast Shock Wave by LS-DYNA
}

\author{
Yan Wang a, Hua Wang, Cunyan Cui, Beilei Zhao, and Tengda Xin \\ Academy of Equipment, Beijing 101416, China. \\ a08wy@163.com
}

Keywords: Boundary condition, blast shock wave, numerical simulation, scaled-distance.

\begin{abstract}
The propagation of TNT blast wave in the air is simulated by using the LS-DYNA finite element analysis software, to study the influence of boundary condition on the overpressure of blast shock wave. In the simulation, three kinds of blast equivalent are selected, which are $5.12 \mathrm{~kg}, 51.2 \mathrm{~kg}$ and 512kg. Angles between four groups of measuring points which are selected and positive X axis are $0^{\circ}, 30^{\circ}, 45^{\circ}, 60^{\circ}$ respectively. In addition, two boundary conditions are selected in the simulation, the zero displacement constraint boundary and the non-reflective boundary. The results indicate that simulation results have better stability when the scaled-distance $Z>1$.2. Secondly, different boundary conditions have different influences on shock wave overpressure, and non-reflective boundary condition will reduce the overpressure value in the vicinity of the boundary. What's more, reasonable selection of measuring points should be emphasized. For example, to select the measurement point of $45^{\circ}$ angle with positive $\mathrm{X}$ axis to obtain the overpressure value is more accurate for a $1 / 4$ cylinder model.
\end{abstract}

\section{Introduction}

In the study of liquid propellant blast hazard analysis and protection, liquid propellant quality is usually converted to the equivalent TNT according to the blast similarity law to predict that of liquid propellant according to the blast law of TNT. The blast of TNT will produce a variety of effects, but the blast shock wave has the strongest destruction and the greatest impact. Therefore, it is significative to study the propagation law for taking effective protection measures.

The experimental method is mainly used to study the blast shock wave in early period. However, it is difficult to study the blast shock wave because of the high risk, high cost and poor reproducibility. With the rapid development of computer technology, the numerical simulation method has become more and more prominent in the study of blast shock wave. Many scholars conduct a lot of studies about the shock wave in different areas by using LS-DYNA finite element analysis software. It is lack of foundation because they set boundary condition according to the experience mostly. In recent years, a few scholars conduct some factors influencing on numerical simulation of blast shock wave. Zhao Beilei has studied the influence of grid density on the simulation accuracy under different equivalents. Gao Xuanneng discovers the influence of the parameters, TNT equivalent, and mesh density on the numerical simulation results is related to the scaled-distance. Yao Chengbao discusses the influence of grid density and non-reflective boundary condition on the calculation results and gives some advice from the perspective of calculating model.

In order to solve the problem that the boundary condition is not clear and the influence of boundary condition on the overpressure is not clear, we will conduct numerical simulation of TNT blast shock wave by LS-DYNA using three different equivalents and under two boundary conditions. It may provide some references for numerical simulation of explosive shock wave though the analysis of the distribution law of overpressure values at different angles and the influence of boundary conditions on shock wave overpressure. 


\section{Numerical Simulation}

\subsection{Establishment of Blast Calculation Model.}

According to the model symmetry, a 1/4 TNT model is established by LS-DYNA to save computing costs and improve efficiency. TNT and air are used as the basic materials in the numerical simulation and cm-g- $\mu$ s is used as the unit system. Element type of TNT and Air is selected eight-node solid unit which is called the three-dimensional SOLID164.

In order to explore the influence of boundary on shock wave overpressure, three kinds of blast equivalent are set to $5.12 \mathrm{~kg}, 51.2 \mathrm{~kg}$ and $512 \mathrm{~kg}$ and two kinds of boundary conditions are conducted for each equivalent and there are six sets of simulation examples, among which Simulation 1, 3, 5 are zero displacement constraint boundary and Simulation 2, 4, 6 are the non-reflective boundary. Four groups of measuring point are selected whose angle with positive $X$ axis are $0{ }^{\circ}, 30^{\circ}, 45^{\circ}, 60^{\circ}$ respectively. The scaled-distance $Z$ ranges from 0.2 to 3 and the interval is 0.2 in each group. The $r$ and $h$ represent radius and height of TNT, while $R$ and $H$ represent radius and height of air. Details of the six simulation models are shown in Table 1.

Table 1. Size parameters of simulation model

\begin{tabular}{cccccc}
\hline Simulation number & $\mathrm{m}_{\mathrm{TNT}} / \mathrm{kg}$ & $\mathrm{R} / \mathrm{cm}$ & $\mathrm{h} / \mathrm{cm}$ & $\mathrm{R} / \mathrm{cm}$ & $\mathrm{H} / \mathrm{cm}$ \\
\hline 1,2 & 5.12 & 10 & 10 & 520 & 30 \\
3,4 & 51.2 & 20 & 25 & 1200 & 300 \\
5,6 & 512 & 50 & 40 & 2410 & 500 \\
\hline
\end{tabular}

As shown in Fig. 1(a) and (b), surface $M$ and the upper and lower surface parallel to the $X O Y$ surface sides are set to non-reflective boundary while surface $S_{1}$ and $S_{2}$ are set to displacement constraints of normal direction. Surface $K$ and $L$ are set to zero displacement constraint and non-reflective boundary at the same time respectively.

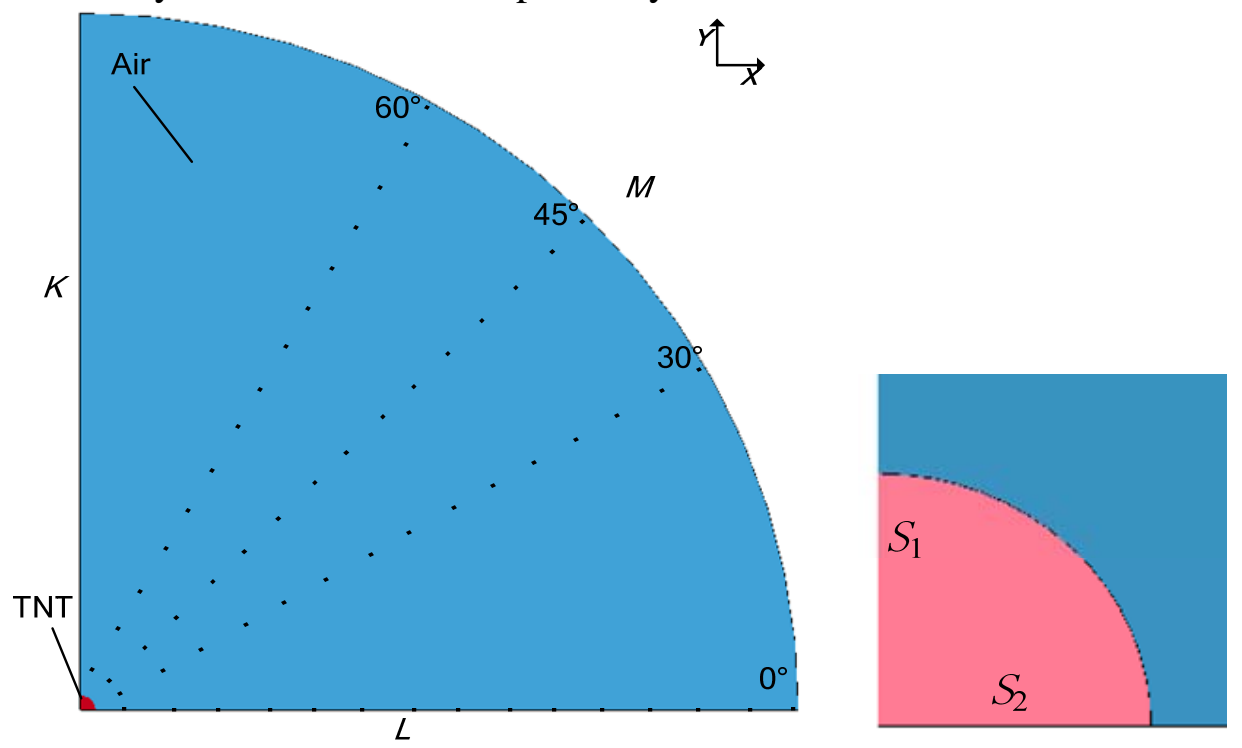

(a)TNT-air model

(b)TNT partially-magnified model

\subsection{Selection of Material Parameters}

Fig. 1 Simulation model

In the simulation process, the air is considered to be the ideal gas without viscosity, and the propagation of the shock wave is isentropic adiabatic process. As is shown in formula (1), *MAT_HIGH_EXPLOSIVE_BURN is selected as material type of TNT and the state equation of JWL (Jones-Wilkins-Lee) is used to describe it.

$$
p=A\left(1-\frac{\omega}{R_{1} V}\right) \mathrm{e}^{-R_{1} V}+B\left(1-\frac{\omega}{R_{2} V}\right) \mathrm{e}^{-R_{2} V}+\frac{\omega E}{V}
$$

In formula (1): $p$ is for pressure. $A, B, R_{1}, R_{2}, \omega$ are parameters of the JWL state equation whose values are determined by experiment. $E$ is the initial internal energy of TNT and $V$ is the current relative 
volume. The parameters of TNT are shown in Table 2, where $\rho_{0}$ is density and $D$ is the detonation velocity.

Table 2. Parameter settings for TNT

\begin{tabular}{cccccccc}
\hline$\rho_{0} /\left(\mathrm{kg} / \mathrm{m}^{3}\right)$ & $D /(\mathrm{km} / \mathrm{s})$ & $A /(\mathrm{GPa})$ & $B /(\mathrm{GPa})$ & $R_{1}$ & $R_{2}$ & $\omega$ & $E /\left(\mathrm{J} / \mathrm{m}^{3}\right)$ \\
\hline 1630 & 6.93 & 371.2 & 3.231 & 4.15 & 0.95 & 0.3 & $7.0 \times 10^{7}$ \\
\hline
\end{tabular}

*MAT_NULL is selected as material type of air and linear polynomial state equation of

*EOS_LNIEAR_POLYNOMIAL is used to describe it, as is shown in formula (2).

$$
P=C_{0}+C_{1} \mu+C_{2} \mu^{2}+C_{3} \mu^{3}+\left(C_{4}+C_{5} \mu+C_{6} \mu^{2}\right) E
$$

In formula (2): $\mu=\rho / \rho_{0}-1, \rho$ and $\rho_{0}$ are for the current density and the initial density respectively. $E$ is the internal energy of the material. $C_{0}, C_{1}, C_{2}, C_{3}, C_{4}, C_{5}, C_{6}$ are parameters of the state equation. The parameters of air are shown in Table 3 , where $V_{0}$ is initial relative volume.

Table 3. Parameter settings for air

\begin{tabular}{cccccccccc}
\hline$\rho_{0} /\left(\mathrm{kg} / \mathrm{m}^{3}\right)$ & $\mathrm{C}_{0} /\left(\mathrm{kg} / \mathrm{m}^{3}\right)$ & $\mathrm{C}_{1} / \mathrm{MPa}$ & $\mathrm{C}_{2}$ & $\mathrm{C}_{3}$ & $\mathrm{C}_{4}$ & $\mathrm{C}_{5}$ & $\mathrm{C}_{6}$ & $E / \mathrm{MPa}$ & $V_{0}$ \\
\hline 1.290 & 1.290 & -0.1 & 0 & 0 & 0 & 0 & 0 & 0.25 & 1.0 \\
\hline
\end{tabular}

\section{Influence of Boundary Condition on Numerical Simulation}

In this paper, the influence of non-reflection boundary on the simulation results of blast wave will be investigated by simulation.

\subsection{Analysis of Shock Wave Overpressure Contours}

When $t$ is $27010 \mu \mathrm{s}$, overpressure contours of the shock wave are shown in Fig. 2 under two types of boundary conditions. We can get different simulation results because the boundary conditions of surface $K$ and $L$ are different. Under the zero-displacement constraint boundary condition, the overpressure distribution is more uniform in all directions at the same distance from the burst source, while overpressure values that are near surface $K$ and $L$ are significantly smaller than the ones away from the boundary. Therefore, the non-reflective boundary surface has a certain effect that reduces the shock wave overpressure value near the boundary on the propagation of the shock wave..

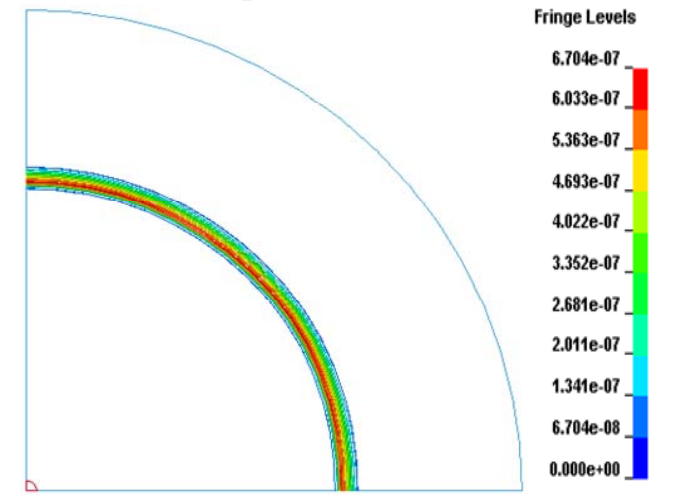

[ al Zero displacement constraint boundary

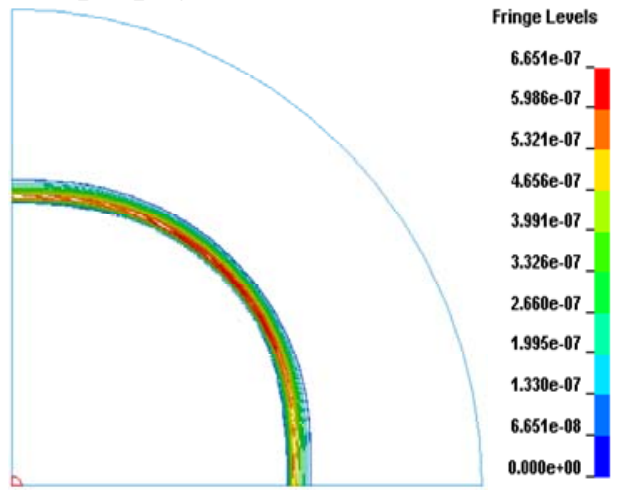

[ b b Non-reflective boundary

Fig. 2 Contour map of shock wave overpressure in two kinds of boundary condition

\subsection{The Distribution Law of Shock Wave Overpressure in Different Measuring Points}

As is shown in Fig. 3, $p$ - $Z$ curves of four group of measurement point were plotted at $0^{\circ}, 30^{\circ}, 45^{\circ}$ and $60^{\circ}$. 


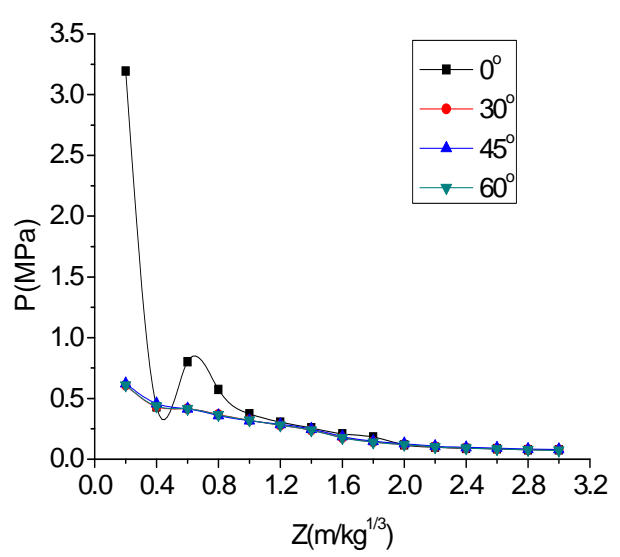

(a)Simulation 1

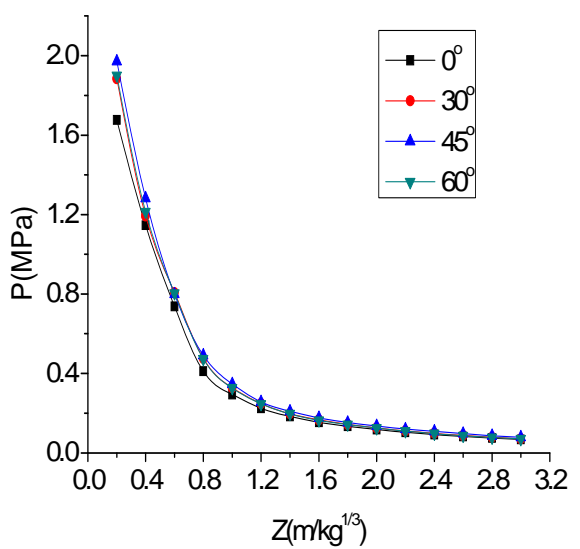

(c) Simulation 3

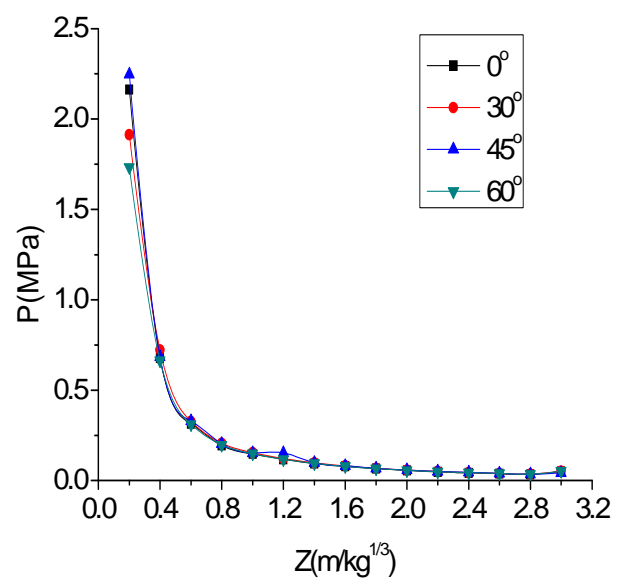

(e)Simulation 5

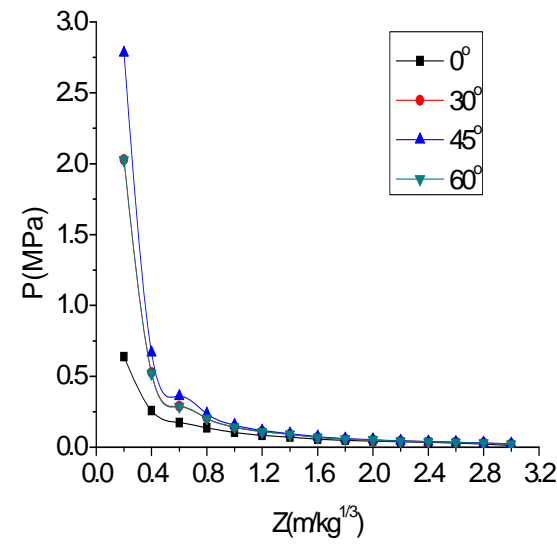

(b) Simulation 2

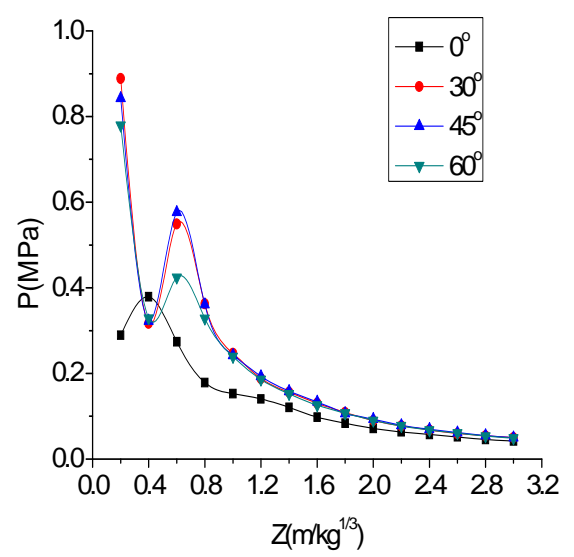

(d) Simulation 4

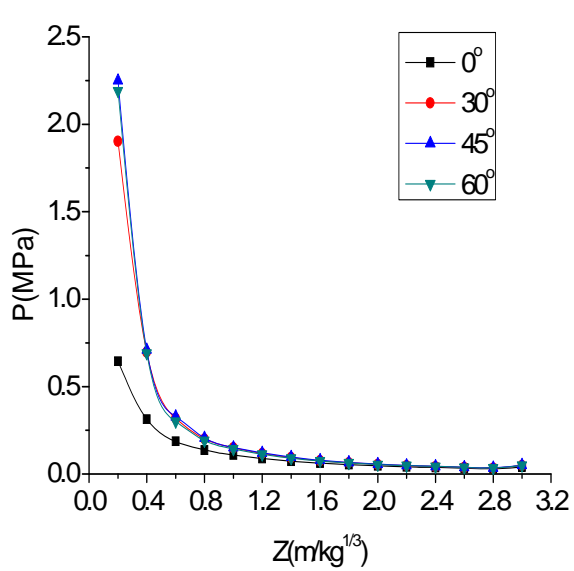

(f) Simulation 6

From fig. 3 we can see that:

Fig. 3 The overpressure varies with the distance

1 ). When the value of $Z$ is small $(Z \leqslant 1.2)$, overpressure values of the four groups of measurement points are quite different from under the same boundary condition. When $Z$ increases gradually $(Z>1.2)$, the overpressure tend to be consistent.

2). By comparing the images $(a)$ and $(b),(c)$ and $(d),(e)$ and $(f)$, we can find that: under the same equivalent, overpressure distribution of the zero-displacement constraint boundary has better consistency than the non-reflective boundary condition for the same scaled-distance. The overpressure of the non-reflective boundary is generally lower than the one of the zero displacement constraint boundary, and the smaller the proportion of the distance, the greater difference between the values. 
3). By comparing the images ( $a),(c),(e)$ and $(b),(d),(f)$, we can see that: when the equivalent and $Z$ are the same, $45^{\circ}$ group has the maximum value of overpressure while $0^{\circ}$ group has the minimum.

\section{Conclusion}

1). Results are more accurate for far field simulation, because simulation results are more stable when $\mathrm{Z}>1.2$.

2). The influence of different boundary conditions is different on the overpressure of shock wave. Further, non-reflective boundary condition will reduce the overpressure in the vicinity of boundary.

3). In order to get more accurate shock wave overpressure value in the simulation analysis, the selection of measuring points should be emphasized. For a 1/4 cylinder model, the measurement point with $45^{\circ}$ angle to positive $\mathrm{X}$ axis is better.

\section{References}

[1]. Peng Mingwei, Chen Xinhua. Analysis of shock wave overpressure for the liquid propellant explosion experiments [J]. Journal of the Academy of Equipment Command and technology. Vol. 12 (2001) No. 4, p. 58-61.

[2]. Yang Xin, Shi Shaoqing, Cheng Pengfei. Prediction and numerical simulation of TNT explosion shock wave overpressure in air [J]. Blasting. Vol. 25 (2008) No. 1, p. 15-16.

[3]. Zhao Beilei, Cui Cunyan, Chen Jingpeng, et al. Influence of Mesh Density on Numerical Simulation Shock Wave Based on LS - DYNA[J]. Automation and instrumentation. (2001) No. 09, p. 227-228+231.

[4]. Wu Xuanneng, Wu Yanjie. Numerical calculation and influence parameters for TNT explosion [J]. Chinese journal of explosives and propellants. (2015) No. 03, p. 32-39.

[5]. Yao Chengbao, Wang Hongliang, Zhang Bohua, et al. Numerical simulation of shock wave generated by TNT explosions in infinite air[J]. Modern Applied Physics.( 2014), No.01, p. 39-44.

[6]. Livermore Software Technology Corporation. LS-DYNA Keyword User's Manual [M]. California: Livermore Software Technology Corporation, 2003, p.2042-2044. 